\title{
S. I.: Pesticides and Veterinary Drugs: Analysis, Environmental Behavior and Risk
}

\author{
Fuwei $\mathrm{Pi}^{1} \cdot{\text { Zongyan } \mathrm{Cui}^{2} \cdot \text { Qiaoying Chang }}^{3} \cdot \mathrm{Pei} \mathrm{Lei}^{4}$
}

Received: 29 June 2021 / Accepted: 20 July 2021 / Published online: 30 July 2021

(c) The Author(s), under exclusive licence to Springer Science+Business Media, LLC, part of Springer Nature 2021

With the requirement of increasing food yields and swift economic growth, the threat posed by human utilized chemical, agricultural and industrial materials have increased over the last several decades. Regardless of the source of these hazards, be it subconscious employing or through misuse, there is little doubt that the persistent/accumulated threats on human health is ever expanding to outpace the range of standards. These hazards mainly include some known and unknown pesticides, veterinary drugs, pathogenic organisms, microbial metabolites, natural toxins and their derivatives. In these times of heightened food security and safety, when faced with the long standing and multiple threats, the unilateral evaluation or detection technology especially for the official standards based on conventional instruments is limited.

This special issue, Pesticides and Veterinary drugs: Analysis, Environmental behavior and Risk, dedicated to current development and trends on pesticides and veterinary drugs for addressing advanced controlling and risk evaluation technologies. We have divided this issue into three sections due to the contributions on the topic: (1) The first section summarized the progress on sensing and removal of pesticides based on 2D nanomaterials or point-of-care-testing (POCT) techniques, and described the representative techniques suitable to rapidly evaluate and simultaneously recognize multiple targets; (2) The second section of this special issue focused on the studies of environmental behaviors, i.e., degradation and sorption with/without control, of some of pesticides and veterinary drugs; (3) The third section of this special issue, which mainly introduced progresses on the scientific risk evaluation, was consistent with the papers that explore residues and derivatives in vegetables and/or fruits, as well as the dietary exposure and health impacts.

The Guest Editors would like to thank Prof. Dr. Erin Bennett, the Editor-in-Chief of Bulletin of Environmental Contamination and Toxicology (BECT), and Prof. Dr. Pang Guofang, the Guest Editor-in-Chief of this special issue, for their kind help and supports. In addition, Dr. Zhong Huan, the Associate Editor of BECT, and Dr. Guangxia Liu, the Assistant Editor of $B E C T$, provided substantial assistance in transforming this effort into reality and we thank their dedicated work. Of course, we are grateful to the authors and the reviewers for their efforts.

Fuwei Pi

pifuwei@jiangnan.edu.cn

1 State Key Laboratory of Food Science and Technology, School of Food Science and Technology, Jiangnan University, Wuxi 214122, China

2 Technology Center of Qinhuangdao Customs, Qinhuangdao 066004, China

3 College of Food Science and Nutritional Engineering, China Agricultural University, Beijing 100083, China

4 State Key Laboratory of Pollution Control and Resources Reuse, School of the Environment, Nanjing University, Nanjing 210023, China 